\title{
Corticosteroids: A controversial therapy for coronavirus disease 2019
}

\author{
Jun She ${ }^{1}$, Dongni Hou ${ }^{1}$, Hong $\mathrm{He}^{2}$, Yuanlin Song ${ }^{1}$ \\ 'Department of Pulmonary Medicine, Zhongshan Hospital, Fudan University, Shanghai, China; \\ 2Department of Anesthesiology, Fudan University Shanghai Cancer Center; Department of Oncology, Shanghai \\ Medical College, Fudan University, Shanghai 200032, China
}

Address for Correspondence: Dr. Yuanlin Song, M.D., Department of Pulmonary Medicine, Zhongshan Hospital, Fudan University, 180 Fenglin Road, Shanghai 200032, China. E-mail: ylsong70@163.com

Dr. Hong He, M.D., Department of Anesthesiology, Fudan University Shanghai Cancer Center; Department of Oncology, Shanghai Medical College, Fudan University, No. 270 Dongan Road, Shanghai 200032, China. E-mail: hyc_hong@163.com

\begin{tabular}{|l|}
\hline Access this article online \\
\hline $\begin{array}{l}\text { Website: } \\
\text { www.intern-med.com }\end{array}$ \\
\hline DOI: \\
10.2478/jtim-2020-0019 \\
\hline Quick Response Code: \\
\hline \\
\hline \\
\hline \\
\hline
\end{tabular}

\section{INTRODUCTION}

Coronavirus disease 2019 (COVID-19) has become a pandemic within months. It is a self-limited viral illness in most cases, but it has spread across the world with rising numbers of deaths. Approximately 10\% patients with COVID-19 required hospital admission, of whom about $10 \%$ were admitted to the intensive care unit (ICU) for acute respiratory distress syndrome (ARDS). ${ }^{[1-4]}$ While COVID-19 appeared to have higher mortality in older male patients with comorbidities, young patients with no comorbidities were also at risk of critical illness including ARDS, multi-organ failure and death. Thus, focus on treatment for severe COVID-19 patients would improve the cure rate and reduce mortality. ${ }^{[5]}$ However, little data is available that describe the pathophysiology of severe COVID-19, and no drug therapies of proven efficacy exist yet.

\section{CYTOKINE STORM}

Among the first reported 41 patients with COVID-19, 32\% (13) patients were admitted to ICU and 15\% (6) died. Compared with severe acute respiratory syndrome (SARS, with increased levels of interleukin [IL]-1 $\beta$, IL-6, IL-12, interferon $[\mathrm{IFN}]-\gamma$, interferon-gammainducible protein [IP]-10, and monocyte chemoattractant protein $[\mathrm{MCP}]-1)^{[7]}$ and middle east respiratory syndrome (MERS, with increased levels of IFN- $\gamma$, tumor necrosis factor [TNF]- $\alpha$, IL-15, and IL-17),${ }^{[8]}$ the patients with COVID-19 had high levels of IL1 $\beta$, IFN $\gamma$, IP10, and MCP1, probably leading to activated T-helper 1 (Th1) cell responses. Furthermore, the patients requiring ICU admission had higher concentrations of granulocyte colony stimulating factor (G-CSF), IP-10, MCP-1, macrophage inflammatory protein 1A (MIP-1A), and TNF- $\alpha$ than those who did not.

Cytokine storm was known as cytokine cascade. It was first proposed by Ferrara in 1993 in graft-versus-host disease, which was an excessive immune response of the body to external stimuli such as viruses or bacteria. ${ }^{[9]}$ There are specific positive feedback mechanisms between cytokines and neutrophils and macrophages. Many cytokines such as TNF- $\alpha$, IL-1, IL-6, IL-8, IL-12, IFN- $\alpha$, IFN- $\beta$, IFN- $\gamma$, and MCP-1 are released rapidly after infection, which is the main cause of ARDS and multiple organ dysfunction syndrome (MODS). COVID-19, SARS, MERS, and H1N1 influenza virus infections could lead to cytokine storm.

On the one hand, cytokine storm plays an important role in ARDS induced by viruses; the levels of IL-6, IL-17, IP-10, MCP-1, and IFN- $\alpha$ increased among patients with severe infection. ${ }^{[10,11]}$ On the other hand, viruses could also interfere with cytokine signaling and contribute to cytokine storm. ${ }^{[12,13]}$ The cytokines such as IL-1 $\beta$, IL- 6 , and TNF- $\alpha$ are responsible for amplifying the inflammatory response, while the cytokines IL-4, IL-10, IL-13, etc. suppress inflammatory responses. Virus clearance depends on inflammatory response, but excessive inflammation caused by uncontrolled cytokine storm induces multiple organ injury. ${ }^{[14]}$

In a retrospective single-center study, the absolute value of lymphocytes decreased 
in most of the 99 patients recruited in Wuhan, China, indicating that like SARS, COVID-19 mainly impaired lymphocytes, especially T lymphocytes. The virus spreads through the respiratory mucosa and infects multiple cell types, induces cytokine storm, generates a series of inappropriate immune responses, and causes alterations in peripheral immune cells such as lymphocytes. Some patients who progressed to ARDS presented cytokine storm and diffuse alveolar damage. ${ }^{[15]}$ Eleven percent patients in the report eventually developed MODS, characterized by elevated creatinine, coagulation disorder, changes in liver enzyme expression, circulatory shock, vascular leakage, and disseminated intravascular coagulation. ${ }^{[16]}$ Therefore, early identification of the cytokine storm and timely treatment of critical cases are of crucial importance.

\section{CORTICOSTEROIDS}

Very limited direct evidence on corticosteroid therapy in patients with COVID-19 has been reported. Indirect evidence from related conditions showed benefits and harms. Corticosteroids are the most commonly used drug therapy to suppress cytokine storm caused by various diseases. Based on limited data, there was no clear benefit and potential harm from corticosteroids; corticosteroid therapy is still controversial.

The World Health Organization guideline for the clinical management of severe acute respiratory infections with suspected COVID-19 recommended that routine corticosteroids should be avoided unless they are indicated for another reason. ${ }^{[17]}$ Carefully designed randomized controlled trials (RCTs) and prospective outcome registries are needed to determine the dose, route, timing, and duration of such treatment on the prevention of clinical deterioration and to better understand the potential harms associated with its use.

\section{Pros}

In a retrospective cohort study including 201 patients with COVID-19-related ARDS in China, treatment with corticosteroids (methylprednisolone) decreased the risk of death (hazard ratio [HR] 0.38, 95\% CI 0.20-0.72). ${ }^{[18]}$ Critically ill patients were more likely to be given corticosteroids in the study. However, administration of corticosteroids was associated with reduced risk of death in patients with ARDS. These findings suggested that corticosteroid treatment may be beneficial for patients with COVID-19 who have developed ARDS. However, the study indicated that the results should be dealt with caution because of the potential bias and residual confounding in this observational study with a small sample size.
Recently, a systematic review and meta-analysis of corticosteroid treatment among ARDS patients included one small cohort study of COVID-19 and seven RCTs of non-COVID-19 patients (risk ratio [RR] 0.72, 95\% CI 0.550.93 , mean difference $17.3 \%$ lesser, low-quality evidence). ${ }^{[19]}$ Corticosteroids were associated with reduced mortality of patients with COVID-19-related ARDS by more than $15 \%$ and reduced the duration of mechanical ventilation. In addition, RCTs in community-acquired pneumonia suggested that corticosteroids may reduce mortality (RR $0.70,95 \%$ CI $0.50-0.98,3.1 \%$ lesser; very low quality evidence) and length of hospital stay (mean difference [MD] 3.6 days shorter, 95\% confidence interval [CI] $0.02-7.2$ days shorter), ${ }^{[20-22]}$ but increase adverse effects of hyperglycemia (risk increase $8.1 \%, 95 \%$ CI $0.7 \%-16.2 \%$ ). ${ }^{[11,23,24]}$ But for COVID-19 patients without ARDS, evidence of very low quality from two observational studies showed increase in mortality associated with corticosteroids (HR $2.30,95 \%$ CI $1.00-5.29$, mean difference $11.9 \%$ greater), which was in line with several observational studies of influenza. Another retrospective cohort study reported that short-term and low-dose treatment of corticosteroids among non-severe COVID-19 patients during the stage of clinical deterioration may possibly prevent disease progression, while having a negligible impact on the viral clearance. ${ }^{[25]}$

The indirect evidence from SARS and MERS should be considered. A systematic review ${ }^{[2]}$ reported 15 studies, 13 of which were inconclusive on the benefits of corticosteroids at the early stage of the disease. One RCT reported delayed viral clearance associated with corticosteroid use in SARS. A study of corticosteroid treatment during the SARS outbreak indicated that no benefit was found for corticosteroids prescribed early in the disease process and might lead to delayed viral clearance.

Recently, an inspiring RCT showed that dexamethasone at a dose of $6 \mathrm{mg}$ once daily for up to 10 days resulted in onethird lower 28-day mortality than usual care in COVID-19 patients who were receiving invasive mechanical ventilation or oxygen; but no benefit and possible harm were found in mild patients who were not receiving respiratory support. This study may have profound effects on the recommendations of corticosteroids in COVID-19 patients. ${ }^{[27]}$

\section{Cons}

A systematic review of observational studies of corticosteroids administered to patients with SARS reported no survival benefit and possible harms (avascular necrosis, psychosis, diabetes, and delayed viral clearance). ${ }^{[28]}$ A study of patients receiving corticosteroids for MERS found no effect of corticosteroids on mortality but delayed 
lower respiratory tract clearance of MERS-CoV. ${ }^{[29]} \mathrm{A}$ systematic review of observational studies of influenza found a higher risk of mortality and secondary infections with corticosteroids; the evidence was judged as very low to low quality due to confounding by indication. ${ }^{[30]}$ A subsequent study that addressed this limitation by adjusting for time-varying confounders found no effect of corticosteroids on mortality. ${ }^{[31]}$

Generalizing evidence from ARDS studies to viral lung injury is problematic because these trials typically include a majority of patients with ARDS of non-pulmonary or sterile cause. ${ }^{[32]}$ A review of treatments for ARDS of any cause, based on six studies with a total of 574 patients, concluded that insufficient evidence existed to recommend corticosteroid treatment. ${ }^{[33]}$ Russell et al. commented that the benefits derived from corticosteroids in the treatment of respiratory infection due to respiratory syncytial virus (RSV), influenza, SARS, or MERS were limited. The available observational data suggested increased mortality and secondary infection rates in influenza infection, impaired viral clearance in SARS and MERS, and increased rates of complications due to corticosteroid therapy in survivors. They concluded that corticosteroid treatment should not be used for the treatment of COVID-19 induced lung injury or shock outside of a clinical trial. ${ }^{[34]}$

\section{Recommendation}

In practice, clinicians tend to use corticosteroids in critical disease. The selective bias in the observational study may result in an increase in mortality among the corticosteroidtreated group. Up until now, clinical evidence was not convincing to recommend against corticosteroids for COVID-19.

According to "The diagnosis and treatment of COVID-19 (version 7)" and "Expert consensus on the use of corticosteroid in patients with 2019-nCoV pneumonia" by National Health Commission and Chinese Thoracic Society, ${ }^{[35,36]}$ if the patient's dyspnea and chest imaging progresses, oxygen saturation significantly decreases, CRP increases, and conventional treatment could not prevent the progress of the disease, which indicate cytokine storm, low-dose and short-term ( $3-5$ days) use of corticosteroids (methylprednisolone $1-2 \mathrm{mg} / \mathrm{kg}$ per day) is recommended. However, the timing of corticosteroid use is essential. The alterations of lymphocyte, CRP, oxygenation index, and imaging manifestations should be considered carefully. After improvement, the dose should be reduced slowly until it is stopped.

Corticosteroids should be administered with caution among patients who have the following conditions: (1) diabetes and receiving oral medication or insulin treatment;
(2) allergy for methylprednisolone, hydrocortisone, dexamethasone, or other corticosteroids; (3) refractory hyperglycemia; (4) epilepsy or delirium; (5) glaucoma; (6) active gastrointestinal bleeding in the past 3 months; (7) hypokalemia; (8) secondary bacterial or fungal infection; (9) immunosuppressive status (e.g., HIV infection within 1 month after chemotherapy, radiotherapy, or surgery); and (10) severe lymphopenia (peripheral blood lymphocytes $<300 / \mu \mathrm{L}){ }^{[37]}$

It should be noted that when more corticosteroids are used, the adverse events are worse. A previous study of 1137 SARS cases from 10 trials showed that higher cumulative dose and longer duration of steroid treatment in SARS patients were more likely to cause osteonecrosis. ${ }^{[37]}$

\section{CONCLUSION}

Many severe COVID-19 patients die from complications such as ARDS and MODS caused by cytokine storm. There is no specific treatment for cytokine storm in clinical practice so far. Corticosteroids are the most commonly used therapy to suppress cytokine storm in various diseases. Based on limited data, corticosteroid therapy for severe COVID-19 is still controversial. Short-course and lowdose applications of corticosteroids in severe COVID-19 patients during the stage of clinical deterioration were recommended. Corticosteroids combined with antiviral therapy, targeted immunotherapy, and traditional Chinese medicines may reduce mortality of severe COVID-19 patients. In addition, active prevention and treatment of complications and respiratory and circulation support are very important measures in the treatment of severe patients with COVID-19.

\section{Source of Funding}

This work was sponsored by Shanghai Municipal Key Clinical Specialty (shslczdzd02201), National Natural Science Foundation (81770075, 82041003), and Shanghai Science and Technology grant (20411950402).

\section{Conflict of Interest}

All authors declare that they have no conflict of interest.

\section{REFERENCES}

1. Guan WJ, Ni ZY, Hu Y, Liang WH, Ou CQ, He JX, et al. Clinical Characteristics of Coronavirus Disease 2019 in China. N Engl J Med 2020; 382: 1708-20.

2. World Health Organization. Coronavirus disease 2019 (COVID-19) Situation Report - 75. Available from: https://www.who.int/docs/defaultsource/coronaviruse/situation-reports/20200404-sitrep-75-covid-19.pdf. Accessed on Aug. 17, 2020. 
3. Cytel. Global Coronavirus COVID-19 Clinical Trial Tracker. 2020. Available from: https://www.covid19-trials.org/. Accessed on Aug. 17, 2020.

4. Wu Z, McGoogan JM. Characteristics of and Important Lessons from the Coronavirus Disease 2019 (COVID-19) Outbreak in China: Summary of a Report of 72314 Cases from the Chinese Center for Disease Control and Prevention. JAMA 2020, Feb 24. doi: 10.1001/jama.2020.2648.

5. She Jun, Liu Jie, Li Jiaming, et al. Therapy for severe and critical corona virus disease 2019 and healthcare personnel protection. Shanghai Med J 2020; 43; 203-6.

6. Huang C, Wang Y, Li X, Ren L, Zhao J, Hu Y, et al. Clinical features of patients infected with 2019 novel coronavirus in Wuhan, China. Lancet 2020; 395: 497-506.

7. Wong CK, Lam CW, Wu AK, Ip WK, Lee NL, Chan IH, et al. Plasma inflammatory cytokines and chemokines in severe acute respiratory syndrome. Clin Exp Immunol. 2004; 136: 95-103.

8. Mahallawi WH, Khabour OF, Zhang Q, Makhdoum HM, Suliman BA. MERS-CoV infection in humans is associated with a pro-inflammatory Th1 and Th17 cytokine profile. Cytokine 2018; 104: 8-13.

9. Ferrara JL. Cytokine dysregulation as a mechanism of graft versus host disease. Curr Opin Immunol 1993; 5: 794-9.

10. Bermejo-Martin JF, Lejarazu RO, Pumarola T, Rello J, Almansa R, Ramírez $\mathrm{P}$, et al. Th1 and Th17 hypercytokinemia as early host response signature in severe pandemic influenza. Crit Care 2009; 13: R201.

11. Kiselevskiy M, Shubina I, Chikileva I, Sitdikova S, Samoylenko I, Anisimova N, et al. Immune Pathogenesis of COVID-19 Intoxication: Storm or Silence? Pharmaceuticals (Basel) 2020; 13: E166.

12. Felsenstein S, Herbert JA, McNamara PS, Hedrich CM. COVID-19: Immunology and treatment options. Clin Immunol 2020; 215: 108448.

13. Azkur AK, Akdis M, Azkur D, Sokolowska M, van de Veen W, Brüggen $\mathrm{MC}$, et al. Immune response to SARS-CoV-2 and mechanisms of immunopathological changes in COVID-19. Allergy 2020; 75: 1564-81.

14. Villar J, Zhang H, Slutsky AS. Lung Repair and Regeneration in ARDS: Role of PECAM1 and Wnt Signaling. Chest 2019; 155: 587-94.

15. Arabi YM, Balkhy HH, Hayden FG, Bouchama A, Luke T, Baillie JK, et al. Middle East respiratory syndrome. N Engl J Med. 2017; 376: 584-94.

16. Chen N, Zhou M, Dong X, Qu J, Gong F, Han Y, et al. Epidemiological and clinical characteristics of 99 cases of 2019 novel coronavirus pneumonia in Wuhan, China: a descriptive study. Lancet 2020; 395: 507-13.

17. Clinical management of severe acute respiratory infection when novel coronavirus (2019-nCoV) infection is suspected. Available from: https:// www.who.int/csr/disease/coronavirus_infections/case-management-ipc/ en/. Accessed on Aug. 17, 2020.

18. Wu C, Chen X, Cai Y, Xia J, Zhou X, Xu S, et al. Risk Factors Associated With Acute Respiratory Distress Syndrome and Death in Patients With Coronavirus Disease 2019 Pneumonia in Wuhan, China. JAMA Intern Med 2020; 180: 1-11.

19. Ye Z, Wang Y, Colunga-Lozano LE, Prasad M, Tangamornsuksan W, Rochwerg B, et al. Efficacy and safety of corticosteroids in COVID-19 based on evidence for COVID-19, other coronavirus infections, influenza, community-acquired pneumonia and acute respiratory distress syndrome: a systematic review and meta-analysis. CMAJ 2020; 192 : E756-67.

20. Meduri GU, Golden E, Freire AX, Taylor E, Zaman M, Carson SJ, et al. Methylprednisolone infusion in early severe ARDS: results of a randomized controlled trial. Chest 2007; 131: 954-63.

21. Steinberg KP, Hudson LD, Goodman RB, Hough CL, Lanken PN, Hyzy R, et al. Efficacy and safety of corticosteroids for persistent acute respiratory distress syndrome. N Engl J Med 2006; 354: 1671-84.
22. Zhao WB, Wan S, Gu DF, et al. Therapeutic effect of glucocorticoid inhalation for pulmonary fibrosis in ARDS patients. Med J Chin PLA 2014; 39: 741-5.

23. Tongyoo S, Permpikul C, Mongkolpun W, Vattanavanit V, Udompanturak $S$, Kocak M, et al. Hydrocortisone treatment in early sepsis-associated acute respiratory distress syndrome: results of a randomized controlled trial. Crit Care 2016; 20: 329.

24. Villar J, Ferrando C, Martinez D, Ambrós A, Muñoz T, Soler JA, et al. Dexamethasone treatment for the acute respiratory distress syndrome: a multicentre, randomised controlled trial. Lancet Respir Med 2020; 8: 267-76.

25. Hu Z, Lv Y, Xu C, Sun W, Chen W, Peng Z, et al. Clinical Use of ShortCourse and Low-Dose Corticosteroids in Patients With Non-severe COVID-19 During Pneumonia Progression. Front Public Health 2020; 8: 355 .

26. Stockman LJ, Bellamy R, Garner P. SARS: systematic review of treatment effects. PLoS Med 2006; 3: e343.18.

27. RECOVERY Collaborative Group; Horby P, Lim WS, Emberson, JR, Mafham M, Bell JL, Linsell L, et al. Dexamethasone in Hospitalized Patients with Covid-19 - Preliminary Report. N Engl J Med 2020 Jul 17; NEJMoa2021436. doi: 10.1056/NEJMoa2021436.

28. Stockman LJ, Bellamy R, Garner P. SARS: systematic review of treatment effects. PLoS Med 2006; 3: e343.

29. Arabi YM, Mandourah Y, Al-Hameed F, Sindi AA, Almekhlafi GA, Hussein MA, et al. Corticosteroid Therapy for Critically Ill Patients with Middle East Respiratory Syndrome. Am J Respir Crit Care Med 2018; 197: 757-67.

30. Rodrigo C, Leonardi-Bee J, Nguyen-Van-Tam J, Lim WS. Corticosteroids as adjunctive therapy in the treatment of influenza. Cochrane Database Syst Rev 2016; 3: CD010406.

31. Delaney JW, Pinto R, Long J, Lamontagne F, Adhikari NK, Kumar A, et al. The influence of corticosteroid treatment on the outcome of influenza A (H1N1pdm09)-related critical illness. Crit Care 2016; 20: 75.

32. Ashbaugh DG, Bigelow DB, Petty TL, Levine BE. Acute respiratory distress in adults. Lancet 1967; 2: 319-23.

33. Lewis SR, Pritchard MW, Thomas CM, Smith AF. Pharmacological agents for adults with acute respiratory distress syndrome. Cochrane Database Syst Rev. 2019; 7: CD004477.

34. Russell CD, Millar JE, Baillie JK. Clinical evidence does not support corticosteroid treatment for 2019-nCoV lung injury. Lancet 2020; 395 : 473-5.

35. National Health Commission. The recommendation of the Diagnosis and treatment of COVID-19 (version 7). Available from: http://www. nhc.gov.cn/xcs/zhengcwj/202003/46c9294a7dfe4cef80dc7f5912eb1989. shtml Accessed on Aug. 17, 2020.

36. Zhao JP, Hu Y, Du RH, Chen ZS, Jin Y, Zhou M, et al. [Expert consensus on the use of corticosteroid in patients with 2019-nCoV pneumonia. Zhonghua Jie He He Hu Xi Za Zhi] 2020; 43: 183-4.

37. Zhao R, Wang H, Wang X, Feng F. Steroid therapy and the risk of osteonecrosis in SARS patients: a dose-response meta-analysis. Osteoporos Int 2017; 28: 1027-34.

How to cite this article: She J, Hou D, He H, Song Y. Corticosteroids: A controversial therapy for coronavirus disease 2019. J Transl Intern Med 2020; 8: 115-8. 